\title{
ASSISTÊNCIA DE ENFERMAGEM AO INDIVÍDUO VÍTIMA DE VIOLÊNCIA SEXUAL
}

NURSING CARE FOR INDIVIDUALS WHO ARE VICTIMS OF SEXUAL VIOLENCE

ATENCIÓN DE ENFERMERÍA A PERSONAS VÍCTIMAS DE VIOLENCIA SEXUAL

Larissa dos Santos Matos $^{1}{ }^{(0}$, Carlos Antonio Farias Sales Junior $^{2}{ }^{-1}$

\section{RESUMO}

Objetivo: analisar na literatura científica sobre a atuação do enfermeiro no atendimento ao indivíduo vítima de violência sexual. Método: trata-se de um estudo bibliográfico, descritivo, tipo revisão integrativa da literatura, com o levantamento dos artigos completos, nos idiomas português, inglês e espanhol, publicados entre janeiro 2014 a setembro de 2019, nas bases de dados LILACS, MEDLINE, no Portal CAPES, e na Biblioteca Virtual SCIELO. Utilizou-se, como instrumento para a coleta dos dados, um formulário semiestruturado. Apresentaram-se os artigos selecionados em um quadro e os seus resultados foram divididos de acordo com eixos temáticos. Resultados: nota-se que a Enfermagem tem um papel fundamental no atendimento à vítima de violência sexual, porém, falta capacitação profissional para que o atendimento possa ser feito de forma adequada, constituindo-se como uma das grandes dificuldades destes profissionais; logo, a Enfermagem forense é capaz de auxiliar na superação desse obstáculo, pois é uma especialização que muito tem a contribuir com essas vítimas. Conclusão: evidencia-se, a partir desta pesquisa, a necessidade de mais pesquisas voltadas para a violência sexual para com outros gêneros além da mulher e da maior formação profissional dos trabalhadores de Enfermagem que atuam nessa área.

Descritores: Enfermagem Forense; Agressão Sexual; Cuidado de Enfermagem; Capacitação Profissional; Delitos Sexuais; Legislação de Enfermagem.

\section{ABSTRACT}

Objective: to analyze the scientific literature on the role of nurses assisting individuals who are victims of sexual violence. Method: this is a bibliographic, descriptive, integrative literature review, with a survey of the full articles, in Portuguese, English, and Spanish, published between January 2014 and September 2019, in the LILACS and MEDLINE databases, the CAPES Portal, and the SCIELO Virtual Library. A semi-structured form was used as an instrument for data collection. The selected articles were presented in a table, and their results were divided according to thematic axes. 
Results: it is noted that nursing has a fundamental role in assisting the victim of sexual violence. However, there is a lack of professional training so that care can be done properly, constituting one of the great difficulties of these professionals; therefore, forensic nursing can help overcome this obstacle, as it is a specialization that has a lot to contribute to these victims. Conclusion: it is evident, from this research, the need for more research aimed at sexual violence towards other genders besides women and the greater professional training of nursing workers who work in this area.

Descriptors: Forensic Nursing; Sexual Assault; Nursing Care; Professional Training; Sexual Offenses; Nursing Legislation.

\section{RESUMEN}

Objetivo: analizar la literatura científica sobre el rol del enfermero en el cuidado de las personas víctimas de violencia sexual. Método: revisión bibliográfica, descriptiva e integradora de la literatura, a través de la encuesta de artículos completos, en portugués, inglés y español, publicados entre enero de 2014 y septiembre de 2019, en las bases de datos LILACS y MEDLINE, el Portal CAPES y el Biblioteca Virtual SCIELO. Se utilizó un formulario semiestructurado como instrumento para la recolección de datos. Los artículos seleccionados se presentaron en una tabla y sus resultados se dividieron según ejes temáticos. Resultados: se observa que la enfermería tiene un rol fundamental en la atención a la víctima de violencia sexual. Sin embargo, existe una falta de formación profesional para que la atención se pueda realizar adecuadamente, constituyendo una de las grandes dificultades de estos profesionales; por tanto, la enfermería forense puede ayudar a superar este obstáculo, ya que es una especialización que tiene mucho que aportar a estas víctimas. Conclusión: se evidencia, a partir de esta investigación, la necesidad de más investigaciones dirigidas a la violencia sexual en géneros distintos al femenino y la mayor formación profesional de enfermeros que laboran en esta área.

Descriptores: Enfermería Forense; Agresión Sexual; Atención de Enfermería; Capacitación Profesional; Delitos Sexuales; Legislación de Enfermería.

1,2Escola Superior Madre Celeste/ESMAC. Ananindeua (PA), Brasil. 1https://orcid.org/0000-0003$\underline{2542-7687} 2$ 2@https://orcid.org/0000-0002-9700-9305

*Artigo extraído do Trabalho de Conclusão de Curso "Assistência de Enfermagem ao Indivíduo Vítima de Violência Sexual". Escola Superior Madre Celeste/ESMAC, 2019. 


\section{Como citar este artigo}

Matos LS, Sales Junior CAF. et al. Assistência de enfermagem ao indivíduo vítima de violência sexual. Rev enferm UFPE on line. 2021;15(2):e245695 DOI: https://doi.org/10.5205/1981-8963.2021.245695

\section{INTRODUÇÃO}

Sabe-se que a expressão violência tem origem no latim violentia, que significava força e poder, ganhando diferentes conceitos durante os anos e lugares, considerando-se importante, por isso, conceituarmos a violência para se poder analisar, elucidar e entender o ato violento. ${ }^{1}$

Percebe-se que a violência se tornou uma questão de saúde pública, diminuindo a qualidade de vida e aumentando os gastos na saúde de forma individual e coletiva. Sublinha-se, nesse contexto, a violência sexual, que vem ganhando destaque devido ao seu grande índice de casos. ${ }^{2}$ Marca-se a violência sexual pelas tentativas ou práticas sexuais sem consentimento por meio de intimidações, perpetradas por qualquer pessoa, incluindo parceiros íntimos, independentemente de como ou onde ocorreram os fatos. Incluem-se, nessa designação, o estupro, o atentado ao pudor e o assédio sexual. ${ }^{3}$

Enfatiza-se que a violência sexual pode ocorrer em vários contextos de interações sexuais, como o sexo forçado nas relações conjugais ou não conjugais, sexo oral, genital ou anal, seja com o órgão genital ou com objetos, sem a anuência da vítima, pedofilia, incestos abusivos, linguagens sexuais impróprias, material pornográfico de atos sexuais indesejados e exploração sexual. ${ }^{4}$

Constatou-se, no Anuário Brasileiro de Segurança Pública de 2019, que, em 2018, a violência sexual chegou a 66.041 registros, sendo que o sexo feminino predominou entre as vítimas $(81,8 \%)$. Registrouse que a maioria tinha até 13 anos de idade (53,8\%), 50,9\% eram de etnia negra e 48,5\%, branca. Calculou-se que a violência sexual teve um crescimento de 4,1\%, referente ao ano de 2017 e, entre 2017 e 2018, registraram-se 127.585 ocorrências de violência sexual no Brasil. Aponta-se, geralmente, que as violências sexuais se iniciam na infância, mas podem ocorrer em qualquer etapa da vida, independentemente da classe social, idade ou gênero. ${ }^{5}$

Observou-se, em estudos realizados nos Estados Unidos, que 63\% das mulheres e $25 \%$ dos homens relataram ter sofrido algum abuso sexual. Alerta-se, no Brasil, que o número de homens sobe para $35 \%$ e o das mulheres desce para $40 \%$. Ressalta-se que essas porcentagens vão além de números absolutos, representando inúmeras consequências para essas vítimas e para a sociedade. ${ }^{2}$

Verifica-se que o indivíduo com histórico de violência sexual apresenta inúmeras complicações decorrentes do abuso sexual, tanto na vida pessoal como no meio social, expressadas por meio dos conflitos familiares, problemas psicológicos, baixo desempenho escolar ou profissional, tristeza, 
raiva, autoestima prejudicada, depressão, pensamentos suicidas e dificuldade de se relacionar socialmente e sexualmente com outras pessoas. ${ }^{6}$

Considera-se, além de ser um evento traumático, que essa violência ultrapassa a esfera social e se torna uma questão pública. Entende-se que o indivíduo vítima de abuso sexual está mais vulnerável a adquirir Infecções Sexualmente Transmissíveis (IST) e estresse pós-traumático, chegando a cometer tentativas de suicídio. Sabe-se que essas e outras consequências podem surgir em curto ou longo prazo, exigindo-se uma assistência integral e contínua, a fim de se evitar danos futuros. ${ }^{7}$

Nota-se que o atendimento ao indivíduo vítima de violência sexual pode ocorrer em todos os serviços/estabelecimentos integrados ao Sistema Único de Saúde (SUS), de acordo com as necessidades de cada caso. Orienta-se que os serviços de saúde públicos e privados notifiquem as suspeitas e que os casos confirmados de todos os tipos de violência, perpetrada em todos os gêneros, independentemente da idade, sejam obrigatoriamente notificados ao Sistema de Informação de Agravos de Notificação (SINAN). Acrescenta-se que esta notificação é apenas uma forma de se garantir os direitos da vítima, não excluindo a denúncia aos demais órgãos. ${ }^{8}$

Salienta-se, também, que o sistema de Vigilâncias de Violências e Acidentes (VIVA) faz parte do SINAN, ou seja, a notificação do VIVA é feita por meio do SINAN. Compõe-se o VIVA por dois itens: o VIVA contínuo, que é praticado por meio do SINAN e integra a vigilância constante das mais variadas formas de violência, incluindo a violência sexual, e o VIVA inquérito, que trata das violências registradas em serviços de urgência e emergência de alguns municípios e é realizado a cada três anos. 9

Baseia-se a notificação no ato de se integrar os órgãos competentes sobre a suspeita ou ocorrência de violência sexual, proporcionando o seu uso na formulação de políticas e ações governamentais para se calcular e reduzir a quantidade de casos. ${ }^{10}$

Torna-se importante que seja trabalhado o atendimento à vítima de violência sexual entre as equipes de saúde, bem como o acolhimento que deve ser feito em todas as etapas e por todos os profisionais de saúde que entrem em contato com a vítima, o sigilo profissional e o protagonismo da vítima são questões que devem sempre ser debatidas para que se possa, assim, oferecer um atendimento de qualidade à vítima. ${ }^{11}$ Requer-se que a equipe seja capacitada para lidar com essas situações, reforçando-se as questões éticas e os deveres e responsabilidades públicos. ${ }^{10}$

Compreende-se que o indivíduo vítima de violência sexual chega ao serviço de saúde em busca de apoio, com medo, vergonha e insegurança. Pontua-se, por vezes, que essa vítima se apresenta tímida e hesitante, comparecendo ao serviço de saúde apenas em busca de atendimento às lesões físicas e 
para receber os cuidados gerais. Considera-se importante, nesse momento, que o enfermeiro saiba abordar essa vítima e identifique os possíveis agravos, diante das vastas consequências, assistindo tanto a saúde física como a psicológica, a fim de se evitar que o (a) paciente seja revitimizado (a) no serviço de saúde. ${ }^{7}$

Avalia-se que a equipe da Atenção Primária à Saúde (APS) tem a maior facilidade para identificar os casos de violência sexual, pelo fato de estar mais próxima às vítimas, criando-se um vínculo maior com os pacientes devido à forte interação com a comunidade e ao desenvolvimento de atividades individuais e coletivas com a família e comunidade. Observa-se que a vítima pode ser atendida tanto pelo enfermeiro como pelo psicólogo e/ou assistente social. ${ }^{11}$

Sabe-se que as mulheres são as maiores vítimas da violência sexual, sendo, muitas vezes, mortas dentro de suas casas por parceiros íntimos. Destaca-se, sobre estes casos, que muitos são marcados pela existência prévia de indícios de agressões. Verifica-se, por vezes, que as vítimas são assassinadas após os abusos sexuais, tratadas como se fossem objetos descartáveis. Detectam-se, ainda, a mutilação genital e a destruição da face por conta da violência física. ${ }^{12}$

Enfatiza-se que as crianças também representam uma grande porcentagem entre as vítimas das violências sexuais. Registrou-se, em 2018, que as meninas de até nove anos de idade responderam por $26,8 \%$ dos casos, sendo que o ápice das violências nesse grupo ocorreu aos 13 anos. Percebeu-se, em relação aos meninos, que essa incidência ocorre mais brevemente, em torno dos sete anos de idade. Pontuou-se, lamentavelmente, que $75,9 \%$ das vítimas possuíam algum vínculo com o perpetrador. ${ }^{5}$

Elencam-se, como outro grupo que é alvo da violência sexual, as Lésbicas, Gays, Bissexuais, Travestis e Transexuais (LGBTT). Nota-se que a sociedade evoluiu em vários fatores, porém, ainda existem muitos paradigmas a serem quebrados, já que aqueles que se mostram contrários aos padrões da sociedade são vistos de forma preconceituosa, tornando-se alvo de violência por conta da sua orientação sexual, por exemplo. ${ }^{13}$

Considera-se importante que esse registro seja realizado, pois, por meio dele, é possível calcular a média da quantidade de casos e se comprovar que o dever dos profissionais está sendo feito, em parceria com os demais órgãos, para se garantir a proteção e a recuperação das vítimas. ${ }^{14}$

Constata-se, infelizmente, que, em alguns lugares do mundo, incluindo o Brasil, não se fornece a assistência adequada para essas vítimas. Apontou-se, em estudos, que as mulheres que sofreram algum tipo de violência sexual têm mais problemas psicológicos do que aquelas que não sofreram violência sexual, porém, enfrentam outro problema de saúde. ${ }^{15}$ 
Alerta-se que não se explicita, no Código de Ética dos Profissionais de Enfermagem (CEPE), sobre a obrigação de se notificar os casos de violência sexual, porém, o CEPE deixa claros os direitos e deveres relativos a essas situações, sendo que os enfermeiros devem fornecer uma assistência livre de danos de negligências, imprudência ou imperícia. Elencam-se, também, as suas proibições profissionais, como ser complacente e provocar e/ou ser negligente com os casos de violência, ou seja, os atos de deixar de notificar ou negligenciar um caso de violência vão contra os seus deveres éticos, podendo levar à perda do direito de exercer a profissão. ${ }^{16}$

Defende-se que os profissionais de saúde, sobretudo, o enfermeiro, são de fundamental importância para a proteção da vítima, pois é este profissional que oferece os primeiros cuidados e, além de exercer as ações necessárias na saúde, pode ser um mediador da vítima com a Justiça, no caso da especialidade da Enfermagem Forense. ${ }^{17}$

Faz-se necessário saber identificar quando o indivíduo está preparado para retomar a sua vida normal ou se ainda precisa de acompanhamento por mais tempo para que possa ser oferecido um atendimento de qualidade a essa vítima. Considera-se ideal que esta seja atendida, primeiramente, por profissionais de saúde que tenham instruções e/ou a capacitação suficiente para se fornecer a melhor assistência possível. ${ }^{18}$

Revelou-se, em um levantamento feito no SINAN, a partir de 172 fichas de adolescentes que sofreram violência sexual, que 70 apresentaram estresse pós-traumático e 15 destes registraram gravidez, porém, $31,4 \%$ das 172 fichas não estavam com estes campos preenchidos, dificultando o levantamento de dados. ${ }^{19}$

Encontram-se falhas na notificação por parte dos profissionais, que acarretam prejuízos para a saúde das vítimas e para o sistema de saúde, pois são o correto preenchimento e a efetivação dessa notificação que fazem com que as devidas providências em relação ao atendimento dessa vítima sejam tomadas para que, assim, as consequências da violência possam ser reduzidas, além de se contribuir para a criação de políticas públicas. ${ }^{19}$

Destaca-se a Lei $n^{\circ} 12.845$, que entrou em vigor no ano de 2013, garantindo-se o atendimento integral e multiprofissional às vítimas de violência sexual em todos os hospitais do SUS, pretendendose sanar os danos físicos e psicológicos. Percebe-se, porém, que só têm sido contemplados os danos físicos como os possíveis ferimentos, sendo preciso mais do que atender a essas necessidades para se fornecer uma assistência de qualidade, o que leva a refletir sobre a importância de ter uma especialização que saiba lidar com essas situações. ${ }^{15}$ 
Sabe-se que a Enfermagem Forense é uma especialidade que une vários conhecimentos específicos para o atendimento de vítimas de violência ${ }^{17}$, enfatizando-se que o enfermeiro, geralmente, é o primeiro contato desta vítima, logo, este profissional que lida com os aspectos $\operatorname{clínicos}^{20}$, o enfermeiro forense, também lida com os aspectos criminais, como as provas, que podem colaborar para se obter a verdade para que essa vítima tenha a justiça alcançada. ${ }^{17}$

Acrescenta-se que os Examinadores de Enfermagem de Agressão Sexual (SANE) e os Examinadores Forenses de Agressão Sexual (SAFE) recebem capacitação e formação clínicas que incluem a coleta de provas, cadeia de custódia, métodos de detecção de lesões e identificação das necessidades da vítima, práticas que colaboram para a qualidade da assistência oferecida e das provas forenses, bem como para a maior compreensão e aplicação da lei, aumentando-se o número de acusações dos responsáveis ao longo dos anos. ${ }^{21}$

Indicou-se, em pesquisas, que os locais que recorrem ao SANE e/ou ao SAFE na realização de exames forenses, nos casos de vítimas de abuso sexual, contribuem mais nos julgamentos, aumentando os índices de condenação. Aponta-se, nos EUA, que mais de 600 jurisdições contemplam o SANE em conjunto à equipe multidisciplinar. ${ }^{21}$

Afirma-se, tendo em mente que o enfermeiro forense compreende o sistema social, o Poder Judiciário e o sistema de saúde público, bem como o vasto conhecimento das ciências forenses, que estes profissionais poderão colaborar com o Poder Judiciário e entidades policiais e governamentais na análise das lesões forenses, estando aptos para identificar as circunstâncias de violência e instituindo diagnósticos, assim como para concretizar os meios de prevenções terapêuticas, analisando os seus resultados. ${ }^{22}$

Elencam-se, entre as suas competências e atribuições gerais, acolher e criar planos estratégicos para as vítimas de violência e os seus familiares, procedendo-se à assistência com o intuito de se ajudar a Justiça, além de saber reconhecer as lesões de maus-tratos e violência diante de situações diversas. ${ }^{16}$

Nota-se que a Enfermagem lida com essas situações no seu dia a dia, exigindo a qualificação para melhor atender a essas vítimas. Visa-se, assim, por este trabalho, a contribuir para com os profissionais da saúde, principalmente, com os enfermeiros, na formação profissional, expandindo os seus conhecimentos e incentivando o seu aprimoramento, assim como para com a comunidade acadêmica, servindo de base para possíveis pesquisas e a formação profissional, para que, assim, a sociedade possa se beneficiar de um atendimento de qualidade. 
Analisar na literatura científica sobre a atuação do enfermeiro no atendimento ao indivíduo vítima de violência sexual.

\section{MÉTODO}

Trata-se de um estudo bibliográfico, descritivo, tipo Revisão Integrativa da Literatura (RIL), método que propicia a união de dados obtidos de pesquisas primárias, elaboradas por meio de estudos diversos, e que necessita de uma rigorosa e sistemática análise de dados, possibilitando-se, assim, uma revisão da literatura que envolva diferentes formas de metodologia, a fim de se adquirir um vasto conhecimento sobre os estudos anteriores, abrangendo-se dados empíricos e científicos. ${ }^{23}$ Caracteriza-se o estudo, desse modo, como exploratório, oferecendo uma visão mais ampla e uma maior familiaridade sobre o assunto abordado, visando a torná-lo mais acessível a todos. Inclina-se a sua organização para um lado mais flexível, considerando-se diferentes aspectos para, assim, trazer uma melhor compreensão sobre o tema.

Elaborou-se a pergunta norteadora por meio de um estudo teórico sobre como o tema tem sido abordado na literatura, a partir das pesquisas feitas sobre a temática e dos índices da violência sexual no Brasil e no mundo, os seus danos e prejuízos para o indivíduo e para a sociedade, bem como a qualidade da assistência na atenção e investigação ao indivíduo que sofreu violência sexual, questionou-se: Qual a assistência de enfermagem prestada às vítimas de violência sexual?

Justifica-se este trabalho dada a sua relevância para os profissionais da saúde, para a comunidade acadêmica e para a sociedade em geral. Buscou-se analisar, a partir de todos os dados levantados neste estudo, que comprovam o elevado índice de violência sexual, como a literatura contemporânea revela a assistência de Enfermagem prestada às vítimas de violência sexual nos serviços de saúde.

Destaca-se que as bases de dados utilizadas para se realizar o levantamento dos artigos foram a Scientific Electronic Library Online (SciELO), Literatura Latino-Americana e do Caribe em Ciências da Saúde (LILACS), Medical Literature Analysis and Retrieval System Online (MEDLINE), Coordenação de Aperfeiçoamento de Pessoal de Nível Superior (CAPES) e PubMed, utilizando-se descritores que coincidem com o tema da pesquisa. Incluíram-se os artigos completos, originais, de revisão e relatos de experiência, que estavam na língua portuguesa, inglesa ou espanhola, publicados no período de janeiro de 2014 a setembro de 2019. Elencam-se os descritores utilizados nas bases de dados SciELO e LILACS, que estavam em português e foram verificados nos Descritores em Ciências da Saúde (DeCS): Enfermagem Forense; Agressão Sexual; Cuidado de Enfermagem e Capacitação Profissional. Registra-se, em relação às bases de dados CAPS, MEDLINE e PubMed, que os descritores estavam em 
inglês e foram verificados no Medical Subject Headings (MeSH): Forensic Nursing; Sexual Abuse; Nursing Care e Professional Training.

Excluíram-se as teses e dissertações, resumos, manuais e artigos que foram publicados antes de janeiro de 2014 e após setembro de 2019, que não estavam na íntegra ou na língua portuguesa, inglesa ou espanhola, os que não estavam com os textos completos liberados, artigos repetidos e os que pertenciam a outras bases de dados que não foram utilizadas neste trabalho.

Desenvolveram-se as buscas nas bases de dados entre agosto de 2019 e setembro de 2019.

Utilizou-se, como instrumento para a coleta dos dados, um formulário semiestruturado composto pela caracterização dos artigos e perguntas abertas onde constam o tipo de estudo, em qual país foi publicado e o ano de publicação, entre outras informações que foram úteis para a pesquisa, facilitando-se o alcance dos objetivos. Verifica-se que os artigos foram pré-selecionados, excluindose aqueles que não atendiam aos critérios de inclusão, restando apenas os que responderam aos objetivos da pesquisa.

Analisaram-se e discutiram-se os dados coletados com base na literatura contemporânea. Consideraram-se o tipo de pesquisa e o seu ano de publicação para se analisar a qualidade do artigo.

Apresentaram-se os artigos selecionados em um quadro e os seus resultados foram divididos de acordo com eixos temáticos, com o objetivo de organizá-los e de se sintetizar o conteúdo encontrado. Incluíram-se todos os gêneros e faixas etárias encontrados na pesquisa e garantiram-se todos os direitos autorais dos dados coletados. Ressalta-se que não foi necessário submeter o estudo ao Comitê de Ética em Pesquisa, pois a pesquisa não foi feita diretamente com seres humanos, tratando-se de uma RIL.

\section{RESULTADOS}

Sinalizaram-se os resultados a partir do agrupamento dos artigos encontrados nas bases de dados. Realizou-se a junção dos descritores nas bases de dados, localizando-se, inicialmente, 1.092.254 artigos. Aplicaram-se, após esta junção, os critérios de inclusão nos filtros de cada base de dados, determinando-se 2.417 artigos. Realizou-se, após essa primeira seleção, a leitura do título e do resumo de cada artigo, para se verificar a semelhança com a pesquisa, chegando-se, assim, a uma segunda seleção, de 74 artigos. Sucedeu-se a leitura completa de cada artigo, realizando-se o levantamento apenas dos artigos que responderam aos objetivos deste trabalho, os quais foram utilizados na colaboração da pesquisa: um total de 13 artigos, excluindo-se os 61 que não corresponderam à pesquisa. 
Nota-se, na seguinte figura, a apresentação dos dados referentes aos 13 artigos analisados que foram utilizados na construção desta pesquisa (Figura 1).

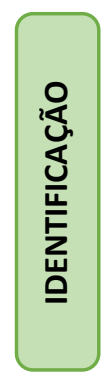

Registros identificados por meio de pesquisas nas bases de dados $(n=1.092 .254)$
Registros identificados por meio de pesquisas em outras fontes de dados $(n=00)$

\section{丕}

Estudos selecionados para a leitura na íntegra $(n=74)$

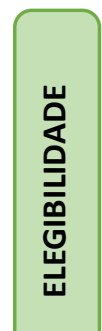

Registros após eliminar os estudos duplicados $(n=2.417)$

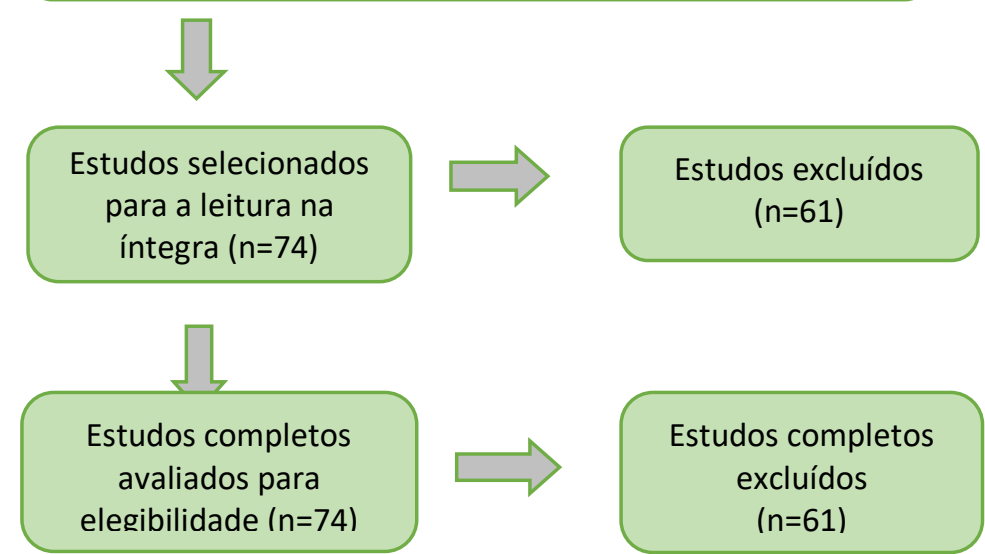

Estudos incluídos em síntese qualitativa $(n=13)$

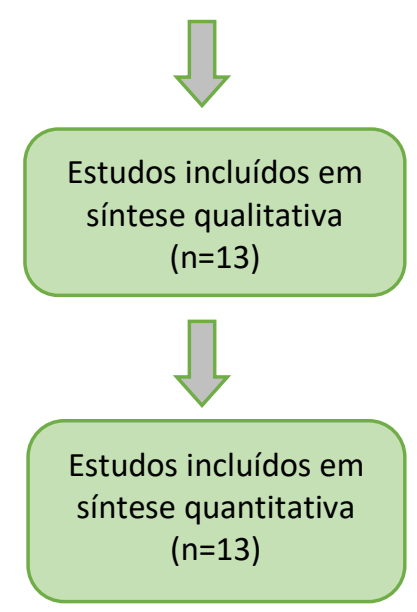

Figura 1. Fluxograma da seleção dos estudos adapatdo do Preferred Reporting Items for Systematic Reviews and Meta-Analyses (PRISMA 2009). Ananindeua (PA), Brasil, 20 de outubro de 2019.

Descrição dos 13 estudos finais analisados, organizados de acordo com o ano de publicação, no período de janeiro de 2014 a setembro de 2019, que foram tuilizados na construçãop desta pesquisa (Figura 2).

\begin{tabular}{|c|lccc|}
\hline$N^{\circ}$ & \multicolumn{1}{|c}{ Título } & País & Ano & Tipo de Estudo \\
\hline 01 & $\begin{array}{l}\text { We desperately need some help here' - } \\
\text { The experience of legal experts with } \\
\text { sexual assault and evidence collection in } \\
\text { rural communities. }\end{array}$ & EUA & 2014 & Qualitativa \\
\hline
\end{tabular}



Hospital-Based Elder Abuse Intervention: literatura An Initial Systematic Scoping Review.

Towards a more integrated and gendersensitive care delivery for victims of sexual assault: key findings and recommendations from the Belgian sexual assault care centre feasibility study.

Vivência de (des)acolhimento por mulheres vítimas de estupro que buscam os serviços de saúde*.

Factors Associated With Forensic Nurses Offering HIV nPEP Status Post Sexual Assault.

Notificação de violência infanto-juvenil em serviços de emergência do Sistema Único de Saúde em Feira de Santana, Bahia, Brasil.

Development of skills-based competencies for forensic nurse examiners providing elder abuse care.

Protocolos na atenção à saúde de mulheres em situação de violência sexual sob a ótica de profissionais de saúde.

Aspectos éticos e legais no cuidado de Enfermagem às vítimas de violência doméstica

Assistência à mulher frente à violência sexual e políticas públicas de saúde: Revisão integrativa.

0 processo de resiliência em mulheres vítimas de violência sexual: uma possibilidade de cuidado*.

Qualificação de profissionais da saúde para a atenção às mulheres em situação de violência sexual.

Establishing Sexual Assault Care Centres in Belgium: health professionals' role in the patient-centred care for victims of sexual violence.

\section{Brasil}

2015

Qualitativa

EUA

Quantitativa

\section{Brasil}

Transversal

Canadá

2016

Quantitativa

Brasil

2016

Qualitativa

Brasil

2017

Qualitativa e

descritiva

Brasil

Revisão da

literatura

Brasil

2017

Qualitativa

Brasil

Qualitativa

Bélgica

2018

Qualitativa

Bélgica

2018

Qualitativa e descritiva 
Figura 2. Descrição dos estudos finais analisados, organizados de acordo com o ano de publicação, no período de janeiro de 2014 a setembro de 2019. Ananindeua (PA), Brasil, 2019.

Destaca-se que o ano de 2015 concentrou o maior número de artigos, sendo quatro pesquisas, representando $30,76 \%$ da amostra, revelando-se que, nesse ano, houve um interesse maior na temática. Ressalta-se que, no ano de 2019, nenhum artigo que correspondesse aos objetivos foi encontrado, sugerindo-se que faltam estudos mais atuais sobre a temática da pesquisa, tendo em vista que, a cada ano, surgem novos avanços científicos e novas leis, sendo necessária a atualização contínua sobre o assunto.

Compreende-se que a maioria dos estudos teve uma abordagem qualitativa $(46,14 \%)$, sendo um nível de evidência baixo. Acrescenta-se que 15,38\% tiveram uma abordagem qualitativa e descritiva e outros 15,38\%, quantitativa. Aponta-se que 15,38\% correspondem a revisões de literatura, encontrando-se apenas um estudo com abordagem transversal (7,69\%).

Constata-se que a maior parte das pesquisas selecionadas foi realizada em mais de um local, ou seja, pesquisas multicêntricas, representando 76,92\% dos artigos, aumentando-se a relevância dos estudos. Registra-se que não houve a identificação do local em 15,38\% das pesquisas, referentes às revisões da literatura, que não abrangem um local específico, e apenas 7,69\% foram realizadas em uma maternidade de referência ao atendimento de vítimas de violência sexual.

Nota-se, em relação à identificação do público-alvo, que a violência sexual ainda é muito centrada na mulher, já que $46,14 \%$ dos trabalhos publicados sobre a violência sexual estão relacionados às vítimas do sexo feminino. Observa-se que $30,76 \%$ das pesquisas não identificaram o seu público-alvo, ou seja, não definiram um gênero para se falar sobre a violência sexual, sugerindo-se que os procedimentos estudados independem do gênero. Aponta-se que os idosos foram alvo de $15,38 \%$ dos estudos e apenas 7,69\% falaram sobre a violência sexual provocada em crianças e adolescentes. Alerta-se que não foi encontrada nenhuma pesquisa sobre a violência sexual relativa exclusivamente ao sexo masculino ou ao grupo LGBT, dado evidenciado na figura 3.

Considera-se de fundamental importância que sejam debatidas as temáticas relacionadas à população LGBT e à população masculina, que são grandes vítimas de violência sexual. 


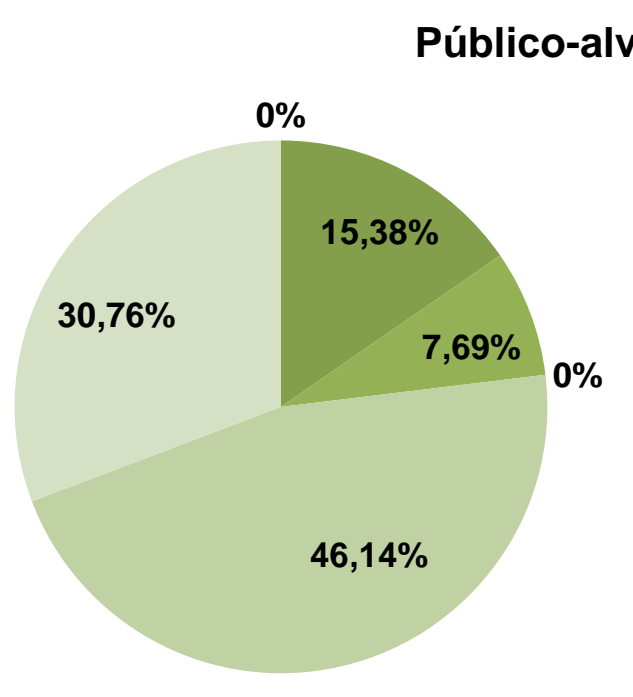

Homem - 0\%

Idoso - 15,38\%

- Infanto-juvenil - 7,69\%

LGBT - 0\%

Mulher - 46,14\%

Não especificado - $30,76 \%$

Figura 3. Representação do público-alvo das publicações. Ananindeua (PA), Brasil,14 de novembro de 2019.

Informa-se que a divisão por eixos temáticos teve como objetivo organizar e sintetizar o conteúdo encontrado. Nota-se que um artigo alcançou mais de um objetivo, logo, contempla mais de um eixo temático.

\section{Assistência de Enfermagem oferecida ao indivíduo vítima de violência sexual}

Identifica-se, no primeiro eixo temático, como se dá a assistência de Enfermagem ao indivíduo vítima de violência sexual.

Constatou-se, nos artigos $02,03,07,11$ e 13, que a atuação do enfermeiro se dá em conjunto à equipe multiprofissional, ou seja, o enfermeiro não está sozinho no atendimento à vítima de violência sexual, trabalhando de forma interdisciplinar.

Revelou-se, nos artigos 07, 11 e 13, que o atendimento à vítima de violência sexual é feito de forma fragmentada. Destacou-se, no artigo 07, que cada profissional lida com as situações de violência sexual de uma maneira diferente e o artigo 13 complementa que a fragmentação do atendimento prejudica a assistência ofertada, além de fazer com que o paciente seja revitimizado nos serviços de saúde.

Verificou-se, nos artigos 03 e 10, que a atuação do enfermeiro se dá por meio de uma postura acolhedora, neutra e compreensiva durante o acolhimento, proporcionando a sensação de proteção e amparo à vítima. Concluiu-se, porém, no artigo 10, que a atuação do enfermeiro nem sempre é realizada da maneira adequada, avaliando-se que alguns agem de forma ética, mas outros não sabem lidar com a situação de forma profissional, pressupondo que a situação vivida foi causada pela própria vítima e que o acontecido não deveria ser considerado abuso, desacreditando o cliente e desvalorizando a sua autonomia, além de ser antiético e contra os seus deveres profissionais. 
Observou-se, nos artigos 05, 08 e 09, que o enfermeiro tem receio de atuar nos casos de violência sexual, seja pela falta de capacitação profissional, pela falta de experiência ou pelo medo de se envolver com o sistema judicial, solicitando, assim, o parecer de outro profissional, encaminhando a vítima para outros serviços ou responsabilizando o sistema de segurança pública.

Alegou-se, no artigo 12, que os enfermeiros atuam na retaguarda de outros profissionais, desenvolvendo uma função de apoio no atendimento agudo e em longo prazo às vítimas de violência sexual.

Percebe-se que os artigos 02 e 09 concordaram entre si e sugeriram que a assistência oferecida pelo enfermeiro se baseia em protocolos, manuais ou diretrizes pré-existentes ou da instituição na qual se trabalha, fornecendo-se as informações pertinentes ao seu atendimento. Relatou-se, no artigo 02, que esses protocolos incluem perguntas já estabelecidas sobre a identificação da violência sexual, relacionadas aos seus sinais, o que facilita a abordagem da vítima. Apontou-se, no artigo 09 , que o enfermeiro se utiliza do Processo de Enfermagem (PE), proporcionando um cuidado individualizado, integral e humanizado, contribuindo para a adesão ao tratamento na recuperação física, psicológica e social e exercendo uma assistência de qualidade e segurança para a vítima, revelando-se, ainda, que há casos de omissão da violência sexual evidenciados pela ausência da notificação.

Ratificou-se esse achado no artigo 08, demonstrando-se que o enfermeiro apenas faz o registro no livro de Enfermagem. Notou-se, no mesmo artigo, que a assistência de Enfermagem respeita os princípios éticos do sigilo e a privacidade da vítima, fornecendo orientações sobre os seus direitos, porém, os profissionais não sabem exatamente a qual órgão encaminhar a vítima de violência sexual. Identificou-se, também, no artigo 03, o respeito pelo sigilo e pela privacidade, por meio do desenvolvimento de uma assistência individualizada, repassando-se as informações sobre os procedimentos que ocorrerão.

Percebeu-se, ainda neste contexto, no artigo 05, que o enfermeiro realiza o encaminhamento da vítima para outros setores, porém, nem sempre é o encaminhamento correto, contribuindo-se para a perpetuação do ciclo de vitimização. Realiza-se, pelo enfermeiro, o preenchimento da ficha de notificação do sistema VIVA.

\section{Dificuldades do enfermeiro no atendimento ao indivíduo vítima de violência sexual}

Efetuou-se, neste eixo, o levantamento das dificuldades encontradas no atendimento de Enfermagem aos indivíduos vítimas de violência sexual. 
Evidenciou-se, nos artigos 05, 06, 07, 08 e 11, que uma das dificuldades encontradas pelo enfermeiro no atendimento ao indivíduo vítima de violência sexual é a falta de formação profissional adequada, relatando-se que, na graduação, não foi abordado o tema ou foi abordado de forma superficial.

Compreendeu-se, nos artigos 07, 08, 09 e 11, que as dificuldades se relacionam à falta de conhecimento sobre o atendimento à vítima de violência sexual e ao desconhecimento sobre as atribuições do enfermeiro, os direitos e os marcos legais da violência sexual. Notou-se, nos artigos 07 e 08, que as enfermeiras desconhecem a ficha de notificação para esse tipo de violência, bem como a sua obrigatoriedade, encontrando dificuldades no seu preenchimento. Perceberam-se, além disso, a insegurança e a falta de habilidades frente aos casos de violência sexual.

Explicaram-se esses dados nos artigos 03, 05 e 12, onde as dificuldades consistiram na falta de especialização na área, de treinamento inadequado e de experiência nos casos de violência sexual.

Salientou-se a falta de recursos físicos, humanos e materiais para o atendimento às vítimas de violência sexual nos artigos 03, 05, 08, 09 e 12. Relataram-se, por alguns profissionais, para se justificar as dificuldades no atendimento, a falta de apoio institucional, da capacitação da equipe, de incentivos, de campanhas, da orientação da equipe e o não repasse de materiais necessários nos artigos 05, 08 e 09.

Avaliou-se que a falta de organização e conhecimento sobre a rede de serviços de saúde disponíveis e a arbitrariedade das informações dificultam o atendimento adequado, dados encontrados no decorrer dos artigos 07 e 09. Adicionou-se, no estudo 09, que o fato de as vítimas procurarem primeiro os serviços de segurança pública dificulta o início da assistência adequada.

Destacou-se, nas pesquisas 05 e 08 , que os profissionais têm dificuldades em lidar com os casos de violência sexual devido ao medo de retaliação por parte do agressor, principalmente, nos casos de violência sexual intrafamiliar. Acrescentaram-se, no artigo 08, o medo de se envolver com um órgão policial e as dificuldades apresentadas pela vítima, como o receio e a vergonha.

Evidenciou-se, pelo estudo 03, a sobrecarga de funções em relação aos profissionais, responsáveis pelo atendimento não só à vítima de violência sexual, mas, também, a outros pacientes. Somou-se, ainda, o baixo número de profissionais em relação à demanda de usuários, que leva a um cuidado superficial.

Elencaram-se, na pesquisa 12, outras dificuldades, como a barreira linguística e a presença dos pais. 
Como a Enfermagem Forense contribui para o atendimento ao indivíduo vítima de violência sexual

Detectaram-se, neste eixo, as contribuições da especialização em Enfermagem Forense para o atendimento ao indivíduo vítima de violência sexual.

Apresentou-se, nos artigos 01 e 04, que a utilização do Examinador de Enfermagem de Agressão Sexual (SANE) é fundamental para o atendimento da vítima de violência sexual. Constatou-se, nos estudos 02 e 03, que os SANE 'S fornecem uma assistência à saúde abrangente, caracterizando-se como um atendimento psicossocial e médico-legal e uma avaliação forense, ambiental e funcional, obtendo-se provas forenses e formulando-se um plano assistencial para a vítima, minimizando-se os danos sofridos e reduzindo-se a probabilidade da vitimização futura.

Verificou-se, no artigo 04, que a utilização dos SANE 'S e Examinador Forense de Agressão Sexual (SAFE) contribui para a criação de protocolos que facilitam o atendimento da vítima, pois, por meio dos exames forenses realizados, é possível, por exemplo, criar um padrão para se detectar os riscos de exposição ao vírus Human Immunodeficiency Virus (HIV), para, assim, se saber a qual paciente se deve oferecer a Profilaxia Pós-Exposição Não Ocupacional (nPEP).

Comentou-se, no artigo 01, sobre como os SANE 'S contribuem com a Justiça, defendendo-se que eles são fundamentais para a investigação criminal, pois, por meio do exame forense, podem se obter provas suficientes para se incriminar o agressor. Entende-se que o SANE 'S é minucioso e criterioso, no sentido de se manter a cadeia de custódia, já que, se este trabalho não for feito da maneira correta, provas importantes e definitivas para o caso podem se perder. Avalia-se que o enfermeiro forense também pode ser uma testemunha importantíssima para o julgamento.

Descreveu-se, no artigo 06, que o enfermeiro forense realiza uma avaliação geral da vítima, atendendo às necessidades físicas, psicológicas, sociais e judiciais, e elabora um documento com questões relevantes para a Justiça, gerando-se um registro preciso, oportuno e completo de todas as observações e cuidados fornecidos, incluindo o exame médico forense, resumo do caso, planos de cuidado, acompanhamento e alta, contribuindo para o trabalho da Justiça, além de se testemunhar em tutela e outros procedimentos necessários.

Comprovou-se, na publicação 12 , que o enfermeiro forense contribui para o atendimento médico e psicossocial, enquanto o artigo 13 constatou que o enfermeiro forense contribui auxiliando o médico nas etapas forenses.

\section{DISCUSSÃO}


Destacou-se, nas publicações, que os profissionais de saúde se tornam essenciais e responsáveis por avaliar e prevenir os casos de violência, de modo que não ponham em risco os familiares e indivíduos envolvidos, utilizando-se meios éticos e legais para, assim, se diminuir os casos de violência. ${ }^{10}$

Considera-se importante que sejam trabalhados o atendimento à vítima de violência sexual entre as equipes de saúde, o acolhimento, que deve ser feito em todas as etapas e por todos os profissionais, o sigilo profissional e o protagonismo da vítima, questões que devem sempre ser debatidas entre a equipe para que se possa oferecer um atendimento de qualidade à vítima e para que ela não seja revitimizada no serviço de saúde. ${ }^{11}$

Entende-se, apesar de se falar bastante sobre a violência sexual, que ainda existe uma subnotificação desses casos; por outro lado, a conscientização e a divulgação sobre a violência sexual fazem com que as vítimas tenham mais coragem de relatar o abuso ocorrido. ${ }^{24}$ Evidencia-se a subnotificação por meio dos estigmas sociais e do preconceito, por conta do perfil de cada pessoa, já que cada indivíduo, vítima ou profissional, enfrenta as situações de uma forma diferente. ${ }^{25}$

Sabe-se que existem falhas na notificação por parte dos profissionais e que essas falhas acarretam prejuízos para a saúde das vítimas e para o sistema de saúde, pois são o correto preenchimento e a efetivação desta notificação que fazem com que as devidas providências em relação ao atendimento desta vítima sejam tomadas, para que, assim, as consequências desta violência possam ser reduzidas, além de se contribuir para a criação de políticas públicas. ${ }^{19}$

Revelou-se que os enfermeiros possuem receio de atender as vítimas, seja por falta de conhecimento ou por medo, porém, é válido ressaltar que esta prática fragiliza o processo de atendimento, pois é importante que o enfermeiro realize a identificação e a notificação breve da vítima para que esta tenha acesso às medidas de profilaxia necessárias para a prevenção de possíveis doenças e/ou a interrupção de uma possível gravidez. ${ }^{8}$ Sublinha-se que é necessário que os profissionais estejam capacitados e preparados para tal atendimento. ${ }^{10}$

Alerta-se que não se explicita no CEPE sobre a obrigação de se notificar ou atender aos casos de violência sexual, porém, o código de ética deixa claros os direitos e deveres do enfermeiro. Orientase que estes forneçam uma assistência livre de danos de negligência, imprudência ou imperícia. Reforçam-se as suas proibições profissionais: ser complacente e provocar e/ou ser negligente com os casos de violência, ou seja, os atos de deixar de notificar ou negligenciar um caso de violência vão contra os seus deveres éticos, podendo levar à perda do seu direito de exercer a profissão. ${ }^{23}$ 
Baseia-se a notificação da violência sexual em se integrar os órgãos competentes sobre a suspeita ou ocorrência de violência sexual, proporcionando-se o seu uso na formulação de políticas e ações governamentais para se reduzir tais casos, assim como para se calcular a quantidade de casos. Salienta-se que é dever dos profissionais de saúde notificar os casos de violência assim que tiverem conhecimento sobre eles. ${ }^{10}$

Aponta-se que a notificação pode ser feita no sistema VIVA por meio do SINAN. Sabe-se que o sistema VIVA é composto por dois itens: o VIVA contínuo, que é feito por meio do SINAN, integra a vigilância constante das mais variadas formas de violência, incluindo a violência sexual, e o VIVA inquérito, que trata das violências registradas em serviços de urgência e emergência de alguns municípios e é atualizado a cada três anos. ${ }^{9}$

Torna-se necessário capacitar os profissionais de saúde para lidar com essas situações, reforçandose as questões éticas, os deveres e as responsabilidades públicas, incluindo a temática nas grades curriculares dos cursos da saúde para que, assim, os profissionais possam ter o mínimo de domínio sobre o assunto, ${ }^{10}$ além de se ter uma especialização capaz de lidar com essas situações, como a Enfermagem Forense. ${ }^{15}$

Percebe-se que o indivíduo vítima de violência sexual chega ao serviço de saúde com medo, vergonha e insegurança, apresentando-se, por vezes, tímido e sem querer falar, apenas em busca de atendimento às lesões físicas e para receber os cuidados gerais. Considera-se importante que, neste momento, o enfermeiro saiba abordar essa vítima e possa identificar os possíveis agravos, diante das vastas consequências, assistindo tanto a saúde física como a psicológica. ${ }^{7}$

Pontua-se que o atendimento ao indivíduo vítima de violência sexual pode ocorrer em todos os serviços/estabelecimentos integrados ao SUS, de acordo com as necessidades de cada caso ${ }^{8}$ porém, não existe um atendimento específico para essas vítimas, sendo atendidas em conjunto a outros pacientes e por profissionais não especializados.

Registra-se, normalmente, que a vítima procura, em primeiro lugar, as unidades policiais e as unidades de saúde, tendo preferência pelas policiais. Nota-se que os locais de saúde mais requeridos são os hospitais e as unidades básicas de saúde, às quais vão, muitas vezes, por conta das lesões físicas. Relatam-se, também, como outros meios de ajuda, os advogados e as Organizações Não Governamentais. ${ }^{26}$

Constatou-se que os locais que utilizam o SANE e/ou o SAFE na realização de exames forenses nos casos de vítimas de abuso sexual contribuem mais nos julgamentos, aumentando os índices de condenação. Sabe-se, nos EUA, que mais de 600 jurisdições contemplam a SANE em conjunto à equipe 
multidisciplinar. ${ }^{21}$ Afirma-se, tendo em mente que o enfermeiro forense compreende o sistema social, o Poder Judiciário e o sistema de saúde público, bem como o vasto conhecimento das ciências forenses, que estes profissionais poderão colaborar com o Poder Judiciário e entidades policiais e governamentais na análise das lesões forenses, estando aptos para identificar as circunstâncias de violência e instituindo diagnósticos, assim como para concretizar os meios de prevenções terapêuticas, analisando os seus resultados. ${ }^{16}$

Aponta-se que a Enfermagem Forense lida com os aspectos criminais, como as provas que podem colaborar para se conseguir a verdade, para que esta vítima tenha a justiça alcançada. ${ }^{20}$ Observa-se que os SANEs e SAFEs recebem capacitação e formação clínica, que incluem a coleta de provas, cadeia de custódia, métodos de detecção de lesões e identificação das necessidades da vítima, práticas que colaboram na qualidade da assistência ofertada. ${ }^{21}$

Elencam-se, entre as suas competências e atribuições gerais, acolher e criar planos estratégicos para as vítimas de violência e seus familiares, procedendo-se à assistência com o intuito de se ajudar a Justiça. Nota-se que, além de saber identificar lesões de maus-tratos e violência, assim como reconhecê-las diante de situações diversas e promover a proteção dos direitos humanos da vítima, familiares e agressores, estes profissionais também desempenham funções como peritos judiciais. ${ }^{16}$

\section{CONCLUSÃO}

Os resultados obtidos neste trabalho foram satisfatórios, visto que após a análise da literatura científica brasileira e estrangeira de um periodo de cinco anos, alcançaram-se os objetivos desta pesquisa.

Nota-se que são necessárias pesquisas mais atuais sobre a temática, que devem ser constantes, assim como os níveis de evidência, que devem ser mais altos, apesar do fato de que a maioria das pesquisas foi multicêntrica, trazendo uma visão mais ampla sobre o assunto. Confirmou-se, na identificação do público-alvo, que a violência sexual ainda é muito centrada na mulher, com uma carência de publicações sobre a violência sexual na população masculina e LGBT.

Comprovou-se que a assistência de Enfermagem é essencial para o atendimento da vítima de violência sexual, que a Enfermagem trabalha em conjunto à equipe multiprofissional e que existe a falta de comunicação entre a equipe, que acaba atuando de forma fragmentada, prejudicando-se o atendimento à vítima. Percebeu-se uma controvérsia entre os autores: ora a assistência de Enfermagem se dá de forma acolhedora, neutra e compreensiva, fornecendo tranquilidade à vítima, ora o profissional tem medo de atender essas vítimas, seja por falta de capacitação ou por não querer se envolver com o sistema judicial. Nota-se, também, uma falha na notificação desta violência por 
parte destes profissionais e, quando se faz o encaminhamento da vítima, nem sempre é realizado da forma adequada para o caso.

Verificou-se que algumas das principais dificuldades do enfermeiro no atendimento da vítima de violência sexual são a falta de capacitação profissional, a inadequação ou a falta de conhecimento sobre as condutas a serem tomadas nestes casos e, também, a falta de recursos físicos, humanos e materiais para o atendimento apropriado, bem como a falta de apoio institucional.

Observou-se que os profissionais com especialização em Enfermagem Forense são cruciais para o atendimento adequado da vítima de violência sexual e que os locais que utilizam esses profissionais contribuem no julgamento dos perpetradores.

Evidencia-se, a partir deste estudo, a necessidade de mais pesquisas voltadas para a violência sexual para com outros gêneros além da mulher, da maior formação profissional para os trabalhadores de Enfermagem para atuar nessa área, assim como a inclusão dessa temática na graduação, contribuindo-se para a formação de profissionais preparados para atender a essa necessidade.

\section{CONTRIBUIÇÕES}

Informa-se que todos os autores contribuíram igualmente na concepção do projeto de pesquisa, coleta, análise e discussão dos dados, bem como na redação e revisão crítica do conteúdo com contribuição intelectual e na aprovação da versão final do estudo.

\section{CONFLITO DE INTERESSES}

Nada a declarar.

\section{REFERÊNCIAS}

1. Misse M. Violência e teoria social. DILEMAS: Revista de Estudos de Conflito e Controle Social. JAN$\begin{array}{llll}\text { ABR } & 2016 ; & \text { 9(1):45-63. } & \text { Available }\end{array}$

https://revistas.ufrj.br/index.php/dilemas/article/view/7672/6

2. Gaspar RS, Pereira MUL. Trends in reporting of sexual violence in Brazil from 2009 to 2013. Cad Saúde Pública. 2018 Nov; 34(11):e00172617. DOI: 10.1590/0102-311X00172617

3. Barbosa JAG, Souza MCMR, Freitas MIF. Violência sexual: narrativas de mulheres com transtornos mentais no Brasil. Revista Panamericana de Salud Publica. 2015;37(4/5):273-8. Available from: https://scielosp.org/article/rpsp/2015.v37n4-5/273-278/pt/ 
4. Ministério da Saúde (BR), Secretaria de Vigilância em Saúde. Análise epidemiológica da violência sexual contra crianças e adolescentes no Brasil, 2011 a 2017 [Internet]. Brasília: Ministério da Saúde; 2018 [cited 2019 Aug 10]. Available from:

http://portalarquivos2.saude.gov.br/images/pdf/2018/junho/25/2018-024.pdf

5.Fórum Brasileiro de Segurança Pública. Anuário Brasileiro de Segurança Pública 2019 [Internet]. São Paulo: Fórum Brasileiro de Segurança Pública; 2019 [cited 2019 Aug 10]. Available from: http: / /www.forumseguranca.org.br/wp-content/uploads/2019/10/Anuario-2019-

FINAL_21.10.19.pdf

6. Santos MJ, Mascarenhas MDM, Rodrigues MTP, Monteiro RA. Characterization of sexual violence against children and adolescents in school - Brazil, 2010-2014. Epidemiol Serv Saude. 2018 June; 27(2):e2017059. DOI: 10.5123/s1679-49742018000200010

7. Aquino RCA, Passos MNS. Nursing assistance for woman victims of sexual violence in primary carE. Rev Estácio Saúde [Internet]. 2018 [cited 2019 Aug 10]; 7(2):42-7. Available from: http://revistaadmmade.estacio.br/index.php/saudesantacatarina/article/viewFile/4496/47964931

8. Ministério da Saúde (BR). Orientações para notificação e atendimento [Internet]. Brasília: Ministério da Saúde; 2018 [cited 2019 Aug 10]. Available from: http://www.saude.gov.br/vigilanciaem-saude/vigilancia-de-violencias-e-acidentes-viva/vigilancia-de-violencias/orientacoes-para$\underline{\text { notificacao-e-atendimento }}$

9. Ministério da Saúde (BR), Secretaria de Vigilância em Saúde, Departamento de Vigilância de Doenças não Transmissíveis e Promoção da Saúde. Viva: Vigilância de Violências e Acidentes: 2013 e 2014 [Internet]. Brasília: Ministério da Saúde; 2017 [cited 2019 Aug 10]. Available from: http://bvsms.saude.gov.br/bvs/publicacoes/viva_vigilancia_violencia_acidentes_2013_2014.pdf

10. Oliveira BG, Freire IV, Assis CS, Sena ELS, Boery RNSO, Yarid SD. Responsibility of health professionals in the notification of cases of violence. Rev Bioét. 2018 July/Sept; 26(3):403-11. DOI: $10.1590 / 1983-80422018263260$

11. Paraná (Estado), Secretaria de Estado da Saúde do Paraná. Superintendência de Atenção à Saúde. Protocolo para o atendimento às pessoas em situação de violência sexual [Internet]. 2nd ed. Curitiba: Secretaria da Saúde; 2018 [cited 2019 Aug 10]. Available from: http://www.saude.pr.gov.br/sites/default/arquivos_restritos/files/documento/2020-

04/protocolo_apsvs_ultimaversao.pdf

12. Meneghel SN, Margarites AF. Femicide in Porto Alegre, Rio Grande do Sul State, Brazil: gender iniquities in dying. Cad Saúde Pública. 2017 Dec; 33(12):e00168516. DOI: 10.1590/0102-311x00168516 
13. Albuquerque GA, Parente JS, Belém JM, Garcia CL. Psychological violence in lesbians, gays, bisexuals, transvestites and transsexuals in Ceará, Brazil. Saúde Debate. 2016 Apr/June; 40(109):10011. DOI: $10.1590 / 0103-1104201610908$.

14. Batista VC, Back IR, Monteschio LVC, Arruda DC, Rickli HC, Grespan LR, et al. Profile of the notifications on sexual violence. J Nurs UFPE on line. 2018 May; 12(5):1372-80. DOI: 10.5205/19818963-v12i5a234546p1372-1380-2018.

15. Messias PP, Silva JS, Sena ELS, Boery RNSO, Yarid SD. Bioethics and support to women victims of sexual violence: literature review. Acta Bioethica [Internet]. 2016 [cited 2019 Aug 10]; 22(1):91-100. Available from: https: //scielo.conicyt.cl/pdf/abioeth/v22n1/art10.pdf

16. Conselho Federal de Enfermagem. Resolução COFEN n 564/2017 [Internet]. Brasília: COFEN; 2017 [cited 2019 Aug 10]. Available from: http://www.cofen.gov.br/resolucao-cofen-no-

\section{7_59145.html}

17. Machado BP, Araújo IMB, Figueiredo MCB. Enfermagem forense: o que é lecionado na licenciatura de enfermagem em Portugal. Revista de Enfermagem Referência. Coimbra set 2019; 4(22):43-50. DOI: $\underline{\text { http: / /dx.doi.org/10.12707/RIV19028 }}$

18. Acosta DF, Gomes VLO, Oliveira DC, Gomes GC, Fonseca AD. Ethical and legal aspects in nursing care for victims of domestic violence. Texto contexto-enferm. 2017 Aug; 26(3):e6770015. DOI: $10.1590 / 0104-07072017006770015$

19. Justino LCL, Nunes CB, Gerk MAS, Fonseca SSO, Ribeiro AA, Paranhos Filho AC. Sexual violence against adolescents in Campo Grande, Mato Grosso do Sul, Brazil. Rev Gaúcha Enferm. 2015; 36(Spe):239-46. DOI: 10.1590/1983-1447.2015.esp.56820

20. Camilo LSS, Dantas TO, Musse JO, Silva DP, Assis ES. PRESERVAÇÃO DA CENA DE CRIME PELO ENFERMEIRO NO SERVIÇO DE ATENDIMENTO MÓVEL DE URGÊNCIA: UMA REVISÃO INTEGRATIVA. Ciências Biológicas e de Saúde Unit. Aracaju out 2017. 4(2):185-202. Available from: https://periodicos.set.edu.br/index.php/cadernobiologicas/article/view/4602

21. National Institute of Justice. National best practices for sexual assault kits: a multidisciplinary approach [Internet]. Washington: Department of Justice; 2017 [cited 2020 May 01]. Available from: https: / / www.ncjrs.gov/pdffiles1/nij/250384.pdf

22. Conselho Federal de Enfermagem. Resolução COFEN nº 556/2017 [Internet]. Brasília: COFEN; 2017 [cited 2019 Aug 10]. Available from: http://www.cofen.gov.br/resolucao-cofen-no- 
23. Soares CB, Hoga LAK, Peduzzi M, Sangaleti C, Yonekura T, Silva DRAD. Integrative review: concepts and methods used in nursing. Rev Esc Enferm USP. 2014 Jan; 48(2):335-45. DOI: 10.1590/S00806234201400002000020 .

24. Dhokia T, Wani R, Jalvee R, Paprikar S. Sexual assault: current scenario at a referral center in Mumbai. Int J Sci Res [Internet]. 2018 Dec [cited 2019 Aug 10]; 7(12):1308-10. Available from: https://www.ijsr.net/archive/v7i12/ART20193884.pdf

25. Mohanamba M. Violence against women: use technology to protect women or girl in problematic situation. Int J Sci Res [Internet]. 2018 Nov [cited 2019 Aug 10]; 7(11):1865-9. Available from: https: / / www.ijsr.net/search_index_results_paperid.php?id=ART20192800

26. Baragatti DY, Rolim ACA, Castro CP, Melo MC, Silva EM. Critical pathway of women facing violence: an integrative review. Rev Panam Salud Publica. 2019 Mar; 43:e34. DOI: $10.26633 /$ RPSP.2019.34

\section{Correspondência}

Larissa dos Santos Matos

E-mail: larissamatos395@gmail.com

Submissão: $30 / 05 / 2020$

Aceito: 09/04/2021

Copyright $\odot 2021$ Revista de Enfermagem UFPE on line.

(c) EY Este é um artigo de acesso aberto distribuído sob a Atribuição CC BY 4.0 Creative Commons AttributionShareAlike 4.0 International License, a qual permite que outros distribuam, remixem, adaptem e criem a partir do seu trabalho, mesmo para fins comerciais, desde que lhe atribuam o devido crédito pela criação original. É recomendada para maximizar a disseminação e uso dos materiais licenciados. 